Desi gn of a system converting an out put radi at i on of frequency tunabl e gyrotron i nto a gaussi an beam

\begin{tabular}{|c|c|}
\hline 著者 & OGAWA I, I DEHARA T, SABCHEVSK S, KASPAREK W \\
\hline $\begin{array}{l}\text { jour nal or } \\
\text { publ i cat } i \text { on } t i t l e\end{array}$ & I nt er nat i onal Jour nal of El ect roni cs \\
\hline vol une & 87 \\
\hline number & 11 \\
\hline page $r$ ange & $1385-1400$ \\
\hline year & $2000-11$ \\
\hline URL & ht t p: //hdl . handl e. net /10098/1541 \\
\hline
\end{tabular}




\title{
Design of a system converting an output radiation of frequency tunable gyrotron into a gaussian beam
}

\author{
I. OGAWA †ा, T. IDEHARAt, S. SABCHEVSKI $\ddagger$ and \\ W. KASPAREK§
}

A novel design of a versatile quasi-optical system for conversion of gyrotron radiation into collimated gaussian beams is presented and discussed. The proposed system consists of a quasi-optical antenna, two ellipsoidal mirrors and a spatial fiter which truncates the sidelobe radiation. The system is appropriate as a transmission line for frequency tunable gyrotrons operating at $T E_{0 n}$ and $T E_{1 n}$ modes. As an illustration of our approach, we present results which demonstrate the applicability of the developed system for conversion of the radiation generated by the Gyrotron FU IVA. The examples include conversion of four $\mathrm{TE}_{1 n}$ mode outputs (TE $12,170 \mathrm{GHz} ; \mathrm{TE}_{13}, 271 \mathrm{GHz} ; \mathrm{TE}_{14}, 372 \mathrm{GHz} ; \mathrm{TE}_{15}, 472 \mathrm{GHz}$ ) into gaussian-like beams and three $\mathrm{TE}_{0 n}$ modes $\left(\mathrm{TE}_{02}, 223 \mathrm{GHz} ; \mathrm{TE}_{03}, 323 \mathrm{GHz}\right.$; $\mathrm{TE}_{04}, 423 \mathrm{GHz}$ ) into bigaussian-like beams.

\section{Introduction}

High frequency gyrotrons are characterized by their capacity to deliver high powers in the submillimetre wavelength range (Zaytsev et al. 1974, Flyagin et al. 1983, Spira-Hakkarainen et al. 1990, Idehara et al. 1995) and their frequency steptunability due to the alternation of the operating mode (Kreischer and Temkin 1987, Brand et al. 1990 b, Idehara et al. 1998, Nusinovich and Read 1999). For many applications such as plasma scattering measurements (e.g. Woskoboinikow et al. 1983. Terumichi et al. 1984, Fekete et al. 1994, Suvorov et al. 1997) where more intense waves are required, the gyrotrons are the most promising candidates as power sources in this region of the electromagnetic spectrum. Very often the gyrotron output needs to be converted into a gaussian-like beam because the gyrotron generates radiation with $\mathrm{TE}_{m n}$ mode structure, which is far from what is usually required from the radiation power source.

In an earlier paper (Ogawa et al. 1997) we presented a quasi-optical system designed to convert the gyrotron output ( $\mathrm{TE}_{15}$ mode, $354 \mathrm{GHz}$ ) into a gaussianlike beam. In this system, a quasi-optical antenna (Vlasov and Orlova 1974) was used to convert the gyrotron output into a linearly polarized beam. Its far-field consists of sidelobes and a main beam which is similar to a gaussian beam. The

†Faculty of Engineering, Fukui University, Fukui 910-8507, Japan.

+ Research Center for Development of Far-Infrared Region, Fukui University, Fukui 910-8507, Japan.

§Institut für Plasmaforschung, Universität Stutgart, Pfaffenwaldring 31, D-70569 Stuttgart, Germany.

of Corresponding author. e-mail: ogawa@maxwell.apphy.fukui-u.ac.jp 
quality of the produced beam was improved only by focusing the main beam using an ellipsoidal mirror. If we apply this system to other $\mathrm{TE}_{1 n}$ modes, the positions and sizes of the beam waist would undergo changes. For most purposes however, a gaussian beam with constant waist size and constant waist position is required.

Over the years a number of step tunable gyrotrons (Gyrotron FU Series) covering a wide frequency range have been developed at Fukui University (Idehara et al. 1999). For such gyrotrons, systems capable of converting the outputs from a set of operating modes are required. This motivated us to develop a new more versatile transmission line which converts several $\mathrm{TE}_{0 n}$ and $\mathrm{TE}_{1 n}$ mode outputs generated by the gyrotron FU IVA (Idehara et al. 1998) into well-collimated beams. Although the size of the main beam at the far-field region depends on gyrotron output mode, it is possible to treat it as a bigaussian beam with constant waist located at the antenna (Ogawa et al. $1999 \mathrm{c}$ ). Taking advantage of this approach the objective beam is obtained by using a confocal mirror system with a spatial filter. In this system the first mirror converts the beam produced by the quasi-optical antenna into the farfield, while the filter truncates the sidelobes.

The paper is organized as follows. In $\S 2$ we outline briefly the approach used for calculation of the radiation patterns. The gist of the technique for the system design based on the gaussian optics is depicted in $\S 3$. The results from the calculations using the Huygens equation for scalar diffraction are presented in $\S 4$. The paper concludes with a summary of the capabilities of the developed conversion system.

\section{Calculation of radiation patterns}

If the electromagnetic fields produced by a quasi-optical antenna are known, then those at any subsequent point can be obtained by solving the Helmholtz equation together with the corresponding boundary conditions on each mirror. Another equivalent approach is based on the use of the Huygens equation for scalar diffraction (e.g. Kong 1906). As in our earlier works (Ogawa ef al. 1997) we follow the latter technique.

The quasi-optical antenna (figure 1) consists of a circular waveguide (internal radius $a_{\mathrm{w}}=10 \mathrm{~mm}$ ) with a step-cut and a parabolic cylinder reflector (focal length $f_{\mathrm{p}}=15.5 \mathrm{~mm}$ ). This antenna converts the TE ${ }_{1 n}$ output of the Gyrotron FU IVA into a linearly polarized beam. The electric and magnetic fields are parallel to the $x$ - and $y$-directions, respectively.

It is well known that for all modes except $\mathrm{TE}_{11}$, the radiation spreads in a hollow cone with a semiangle given by
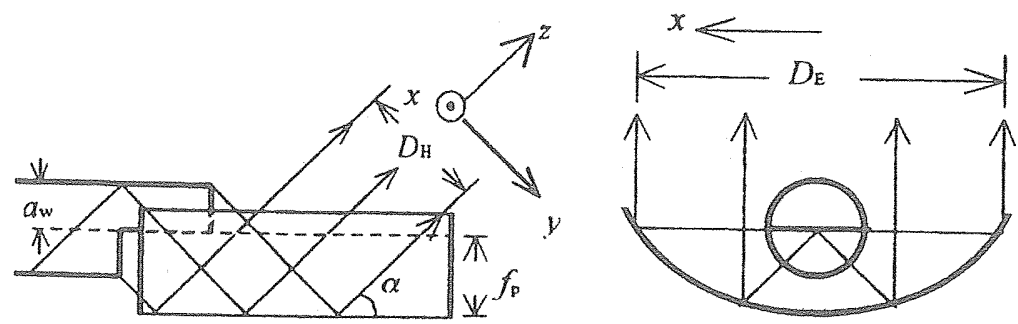

Figure 1. Quasi-optical antenna. 


$$
\sin \alpha=\frac{k_{\mathrm{c}}}{k}
$$

where $k_{\mathrm{c}}=\rho_{m n}^{\prime} / a_{\mathrm{W}}$ is the cutoff wavenumber, $\rho_{m n}^{\prime}$ is the nth root of $J_{m}^{\prime}(\rho)=0, a_{\mathrm{W}}$ is the waveguide radius and $k$ is the wavenumber. In the gyrotron cavity the mode is very close to cutofr and

$$
k=k_{\mathrm{cav}}=\frac{\rho_{m n}^{\prime}}{a_{\mathrm{c}}}
$$

where $a_{c}$ is the cavity radius. If we neglect mode conversion

$$
\alpha=\sin ^{-1} \frac{a_{\mathrm{c}}}{a_{\mathrm{w}}}
$$

This means that to a first approximation the angle $\alpha$ is independent of the mode. In our case, $\alpha=8.63^{\circ}$. The fields over this image source are calculated using the wellknown approach based on geometrical optics (Wada and Nakajima 1986, Brand et al. 1990 a).

The incident electromagnetic fields at the first mirror are calculated using the Huygens equation. The electromagnetic fields reflected from the mirror are given by the boundary conditions (Ogawa et al. 1999 b). The electromagnetic fields on the subsequent mirrors are obtained using repeatedly the Huygens equation and the boundary conditions together with the previously calculated results as the source fields.

\section{Design of the systern using garussian optics}

In order to investigate how the beams spread, their intensity profiles have been calculated for a plane situated far from the image source. The image source is located so that the beam with polarization in the $x$-direction propagates along the $z$-direction (figure 2). The intensity profiles for $T E_{0 n}$ modes in the far-field region are shown in figure 3 and those for $T E_{1 n}$ modes are shown in figure 4.

Each far-field consists of a main beam and additional sidelobes. The main beams for $\mathrm{TE}_{0 n}$ modes have elliptical cross-section and their spot sizes are given by assuming a bigaussian beam whose waist (waist size $w_{x 0}=26.4 \mathrm{~mm}$ in width and $w_{y 0}=18.0 \mathrm{~mm}$ in length) is located at the centre of the image source (table 1) (Ogawa et al. $1999 \mathrm{c}$ ). On the other hand, those for $\mathbf{T E}_{1 n}$ modes are circular and

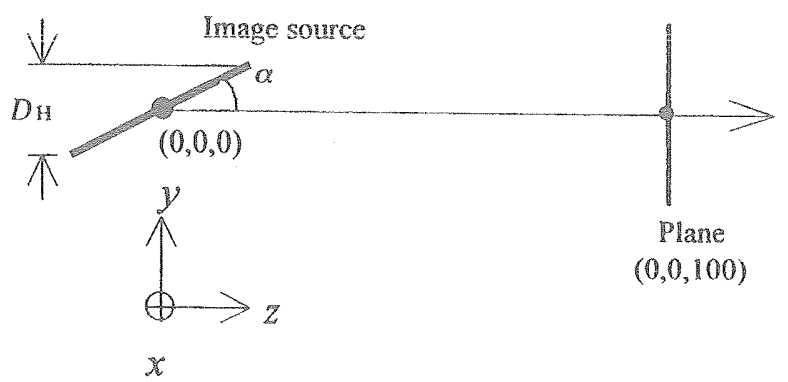

Figure 2. Plane image source used in the calculation of the subsequent radiation patterns of the quasi-optical antenna with a single parabolic reflector. The beam propagates along the $z$-axis. 
(a)

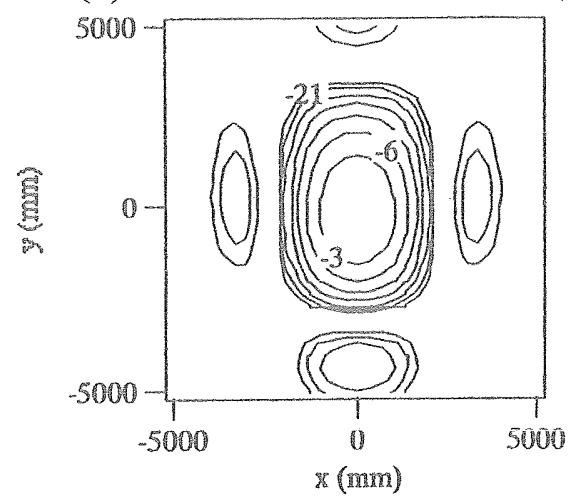

(b)

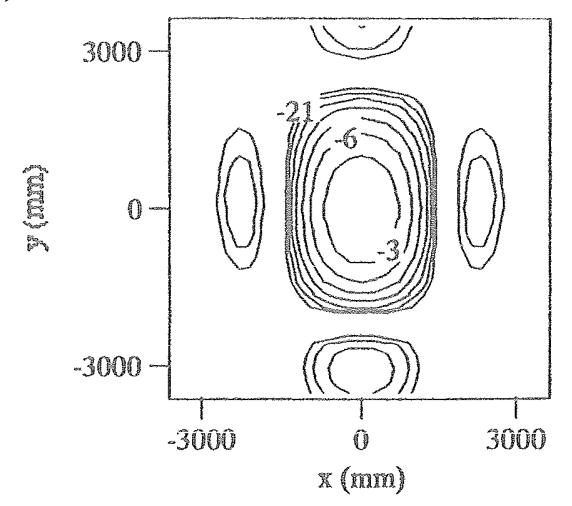

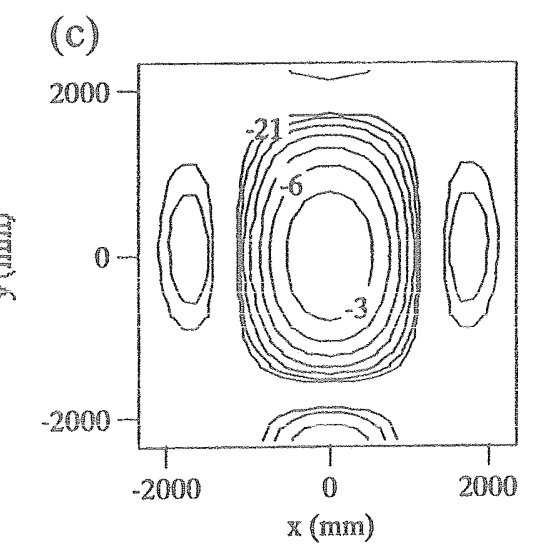

Figure 3. Calculated intensity contours in the far-field region. Distance from the image source is $100 \mathrm{~m}:$ (a) $\mathrm{TE}_{02}$ mode $(223 \mathrm{GHz}) ;(b) \mathrm{TE}_{03}$ mode $(323 \mathrm{GHz})$; (c) $\mathrm{TE}_{04}$ mode $(423 \mathrm{GHz})$. Contours are in decibels relative to the intensity maximum.

their spot sizes are given by assuming a gaussian beam with waist size $w_{x 0}=w_{y 0}=18.1 \mathrm{~mm}($ table 1$)$.

The intensity of the bigaussian beam is given by

$$
I=\frac{2 p_{0}}{\pi w_{x} w_{y}} \exp \left(-\frac{2 x^{2}}{w_{x}^{2}}\right) \exp \left(-\frac{2 y^{2}}{w_{y}^{2}}\right)
$$

where $w_{x}$ and $w_{y}$ are the spot sizes in the $x$-and $y$-directions, respectively and $P_{0}$ is the total beam power. We will defne the spot size of such a beam as the radius of the $-8.69 d 8$ contour, where the intensity is $e^{-2}$ smaller than at the maximum.

propagation and focusing of a bigaussian beam is most conveniently treated by the use of the complex beam parameters $q_{x}$ and $q_{y}$ (e.g. Siegman 1971, Ogawa et al. 1999 a).

In the system under consideration (figure 5), the focal length $f_{1}$ of the mirror ml coincides with the distance $d_{1}$, i.e. $f_{1}=d_{1}=1.5 \mathrm{~m}$. For this special arrangement, the far field of the image source appears in the $y=1.241$ plane (Ogawa et al. 1999 b). 
(a)

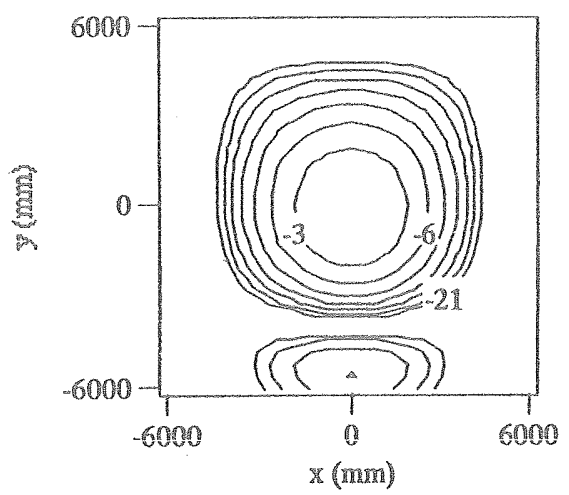

(c)

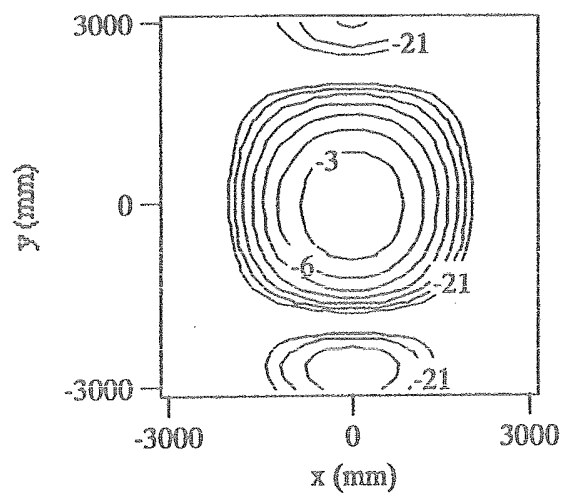

(b)

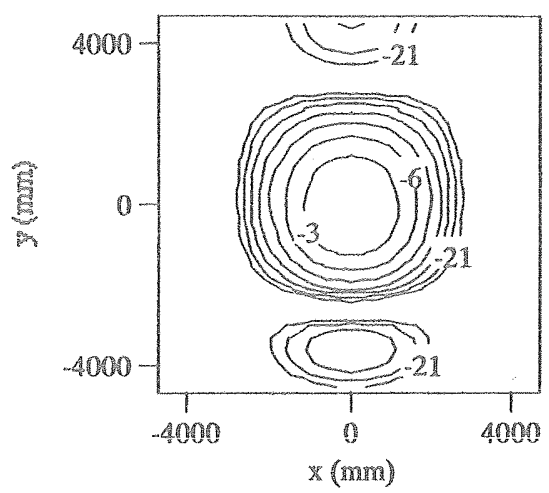

(d)

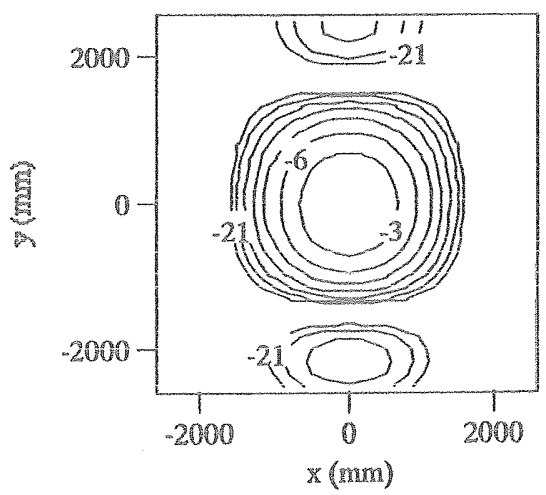

Figure 4. Calculated intensity contours in the far-field region. Distance from the image source is $100 \mathrm{~m}$ : (a) $\mathrm{TE}_{12}$ mode $(170 \mathrm{GHz}) ;$ (b) $\mathrm{TE}_{13}$ mode $(271 \mathrm{GHz})$; (c) $\mathrm{TE}_{14}$ mode $(372 \mathrm{GH} / \mathrm{Z}) ;(d) \mathrm{TE}_{15}$ mode $(472 \mathrm{GHz})$. Contours are in decibels relative to the intensity maximum.

\begin{tabular}{lccccc}
\hline Mode & $\begin{array}{c}\text { Frequency } \\
(\mathrm{GHz})\end{array}$ & $\begin{array}{c}w_{x} \\
(\mathrm{~m})\end{array}$ & $\begin{array}{c}w_{y} \\
(\mathrm{~m})\end{array}$ & $\begin{array}{c}w_{x 0} \\
(\mathrm{~mm})\end{array}$ & $\begin{array}{c}w_{y 0} \\
(\mathrm{~mm})\end{array}$ \\
\hline$T E_{02}$ & 223 & 1.61 & 2.38 & 26.6 & 18.0 \\
$T E_{03}$ & 323 & 1.13 & 1.64 & 26.2 & 18.0 \\
$T E_{04}$ & 423 & 0.85 & 1.25 & 26.5 & 18.0 \\
$T E_{12}$ & 170 & 3.09 & 3.14 & 18.2 & 17.9 \\
$T E_{13}$ & 271 & 1.92 & 1.94 & 18.3 & 18.1 \\
$T E_{14}$ & 372 & 1.40 & 1.42 & 18.3 & 18.0 \\
$T E_{15}$ & 472 & 1.12 & 1.12 & 18.1 & 18.0 \\
\hline
\end{tabular}

Table 1. Spot sizes $w_{x}$ and $w_{y}$ in the far-field region $(z=100 \mathrm{~m})$ of the beam produced by image sources at various gyrotron operating modes and waist sizes $w_{x 0}$ and $w_{y 0}$ of equivalent bigaussian beam having its waist at the image source. 


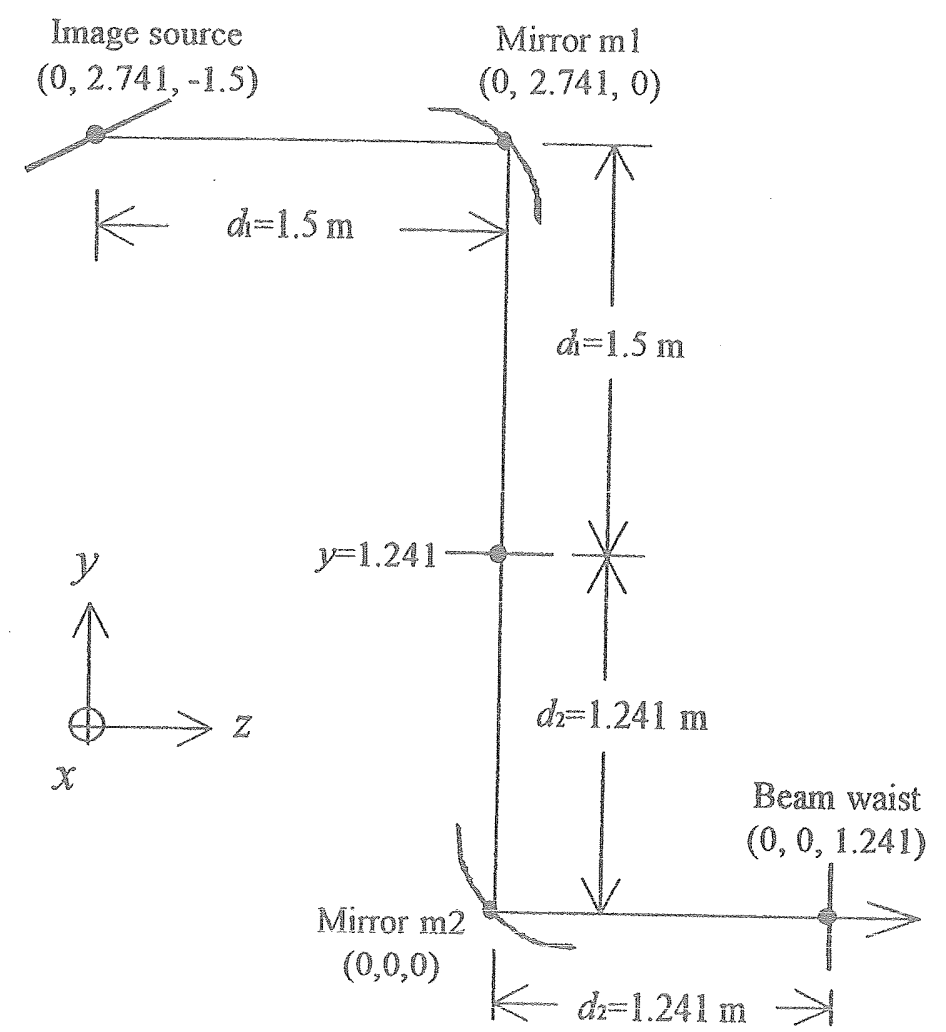

Figure 5. Quasi-optical system.

Treating the main beam as a bigaussian one with a waist at the position of the image source, the beam waist sizes $w_{x 0^{\prime}}$ and $w_{z 0^{\prime}}$ at the $y=1.241$ plane can be obtained from the relations

$$
w_{x 0^{\prime}}=\frac{f_{1} \lambda}{\pi w_{x 0}} \quad w_{z 0^{\prime}}=\frac{f_{1} \lambda}{\pi w_{y 0}}
$$

The equations show that since one waist is at the image source, the beam will have a waist at the $y=1.241$ plane. Because the mirror $m 2$ has also the special arrangement $\left(f_{2}=d_{2}=1.241 \mathrm{~m}\right.$ ), the beam will come to a waist at the $z=1.241$ plane. The final waist sizes $w_{x 0^{\prime \prime}}$ and $w_{y 0}$ at the $z=1.241$ plane are given by

$$
w_{x 0^{\prime \prime}}=\frac{f_{2}}{f_{1}} w_{x 0} \quad w_{y 0^{\prime \prime}}=\frac{f_{2}}{f_{1}} w_{y 0}
$$

This result generally holds for a confocal system consisting of two mirrors. Note that it does not depend on frequency.

The focal lengths $f_{1}$ and $f_{2}$ are determined to be $1.5 \mathrm{~m}$ and $1.241 \mathrm{~m}$, so this system produces a gaussian beam with the waist size $w_{x 0^{\prime \prime}}=w_{y 0^{\prime \prime}}=15.0 \mathrm{~mm}$ for $T E_{1 n}$ mode. It also converts $T E_{0 n}$ mode gyrotron output into a bigaussian beam with the waist sizes $w_{x 0^{\prime \prime}}=21.8 \mathrm{~mm}$ and $w_{y 0^{\prime \prime}}=14.9 \mathrm{~mm}$ for $\mathrm{TE}_{0 n}$ mode. 


\section{Calculation resulls using the Hillyygens enduation}

The mirrors $m 1$ and $m 2$ are wide enough $(\mathrm{ml}$ is $160 \mathrm{~mm}$ in width and $590 \mathrm{~mm}$ in length, while the corresponding dimensions for $\mathrm{m} 2$ are $170 \mathrm{~mm}$ and $240 \mathrm{~mm}$ ) to avoid any difraction losses due to the beam truncation. The shape of the mirror $\mathrm{ml}$ is defined by an ellipse with focal points at $(0,2.741,-10)$ and $(0,0.976,0)$ resulting in a focal length of $f_{1}=1.5 \mathrm{~m}$. Analogously, the shape of the mirror $\mathrm{m} 2$ is defined also by an ellipse with focal points at $(0,10,0)$ and $(0,0,1.417)$ which gives a focal length of $f_{2}=1.241 \mathrm{~m}$.

The intensity profiles at the $y=1.241$ plane for $\mathrm{TE}_{0 n}$ modes are shown in figure 6 and those for $\mathbb{T E}_{1 n}$ modes are presented in figure 7. Remembering that the $x$ - and $z$ directions in figures 6 and 7 correspond to the $x$-and negative $y$-directions in figures 3 and 4 , one can see that the corresponding intensity profiles are similar. The spot sizes $w_{x^{\prime}}$ and $w_{z^{\prime}}$ of the main beam agree well with the waist sizes $w_{x 0^{\prime}}$ and $w_{z 0^{\prime}}$ given by equation (5) (table 2). This corroborates that the radiation pattern produced at the $y=1.241$ plane is the far-field of the image source. As can be seen from the field
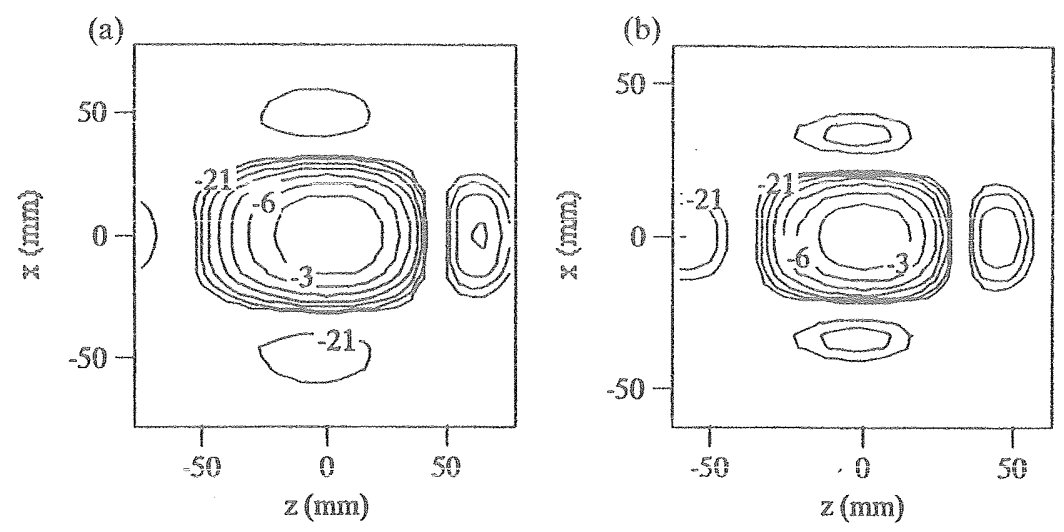

(c)

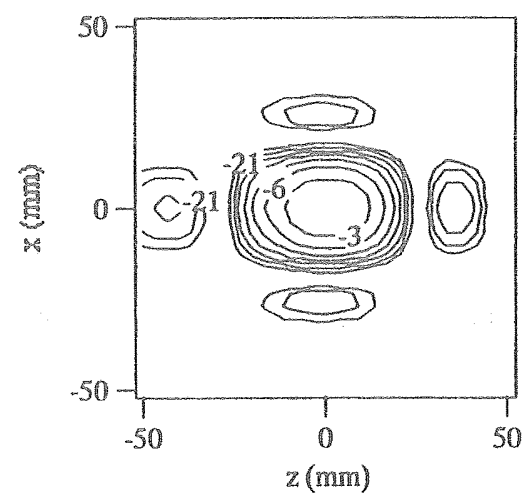

Figure 6. Calculated intensity contours at the $y=1.241$ plane: $(a)$ TE 02 mode $(223 \mathrm{GHz}) ;(b)$ $\mathrm{TE}_{03}$ mode $(323 \mathrm{GHz}) ;(c) \mathrm{TE}_{04}$ mode $(423 \mathrm{GHz})$. Contours are in decibels relative to the intensity maximum. 
(a)

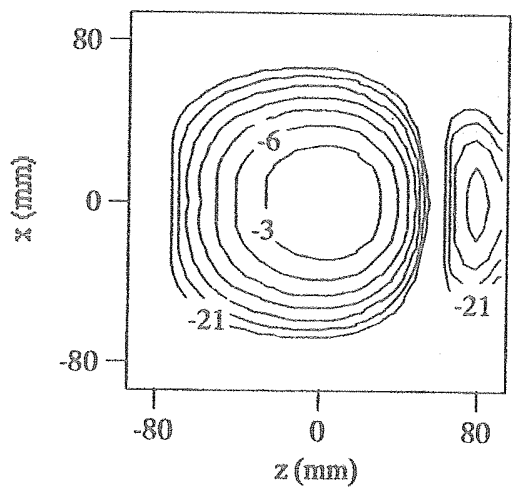

(c)

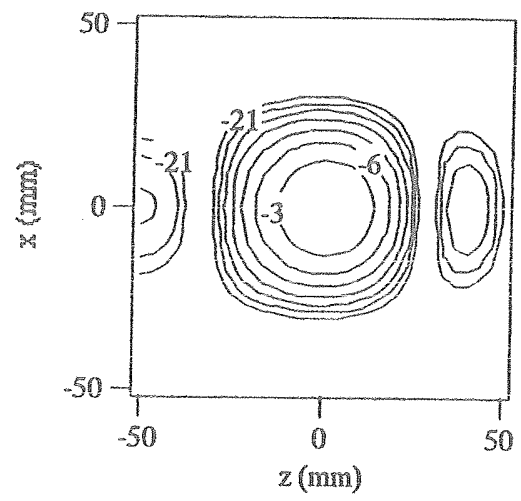

(b)

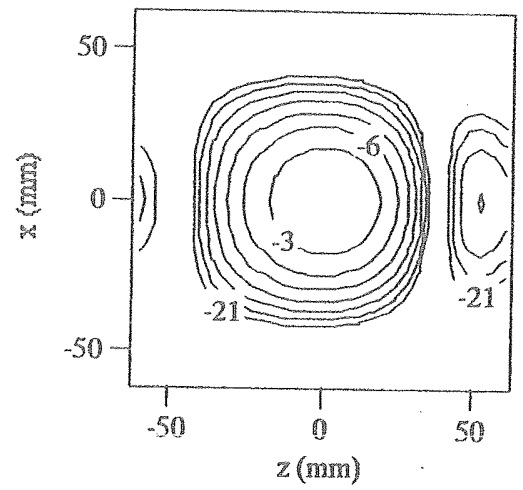

(d)

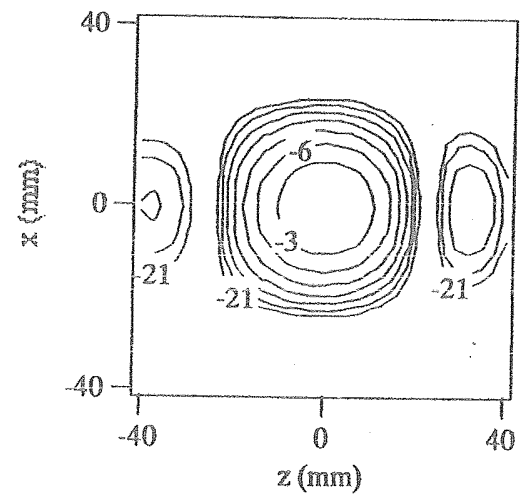

Figure 7. Calculated intensity contours at the $y=1.241$ plane: $(a) \mathrm{TE}_{12}$ mode $(170 \mathrm{GHz}) ;(b)$ $\mathrm{TE}_{13}$ mode $(271 \mathrm{GHz}) ;(c) \mathrm{TE}_{14}$ mode $(372 \mathrm{GHz}) ;($ d $) \mathrm{TE}_{15}$ mode $(472 \mathrm{GHz}$ ). Contours are in decibels relative to the intensity maximum.

\begin{tabular}{|c|c|c|c|c|c|c|c|c|}
\hline \multirow[b]{2}{*}{ Mode } & \multicolumn{4}{|c|}{ Gaussian optics } & \multicolumn{4}{|c|}{ Calculation } \\
\hline & $\begin{array}{c}w_{x 0^{\prime}} \\
(\mathrm{mmm})\end{array}$ & $\begin{array}{c}w_{z 0^{\prime}} \\
(\mathrm{mm})\end{array}$ & $\begin{array}{l}w_{x 0^{\prime \prime}} \\
(\mathrm{mm})\end{array}$ & $\begin{array}{c}w_{y 0^{\prime \prime}} \\
(\mathrm{inm})\end{array}$ & $\begin{array}{c}w_{x^{\prime}} \\
(\mathrm{mm})\end{array}$ & $\begin{array}{c}w_{z^{\prime}} \\
(\mathrm{mm})\end{array}$ & $\begin{array}{c}W_{x}^{\prime \prime} \\
(\mathrm{nmm})\end{array}$ & $\begin{array}{c}w_{y^{\prime \prime}} \\
(\mathrm{mm})\end{array}$ \\
\hline $\mathrm{TE}_{02}$ & 24.3 & 35.7 & 21.8 & 14.9 & 23.9 & 35.6 & 22.3 & 16.3 \\
\hline$T E_{03}$ & 16.8 & 24.6 & 21.8 & 14.9 & 17.0 & 25.0 & 24.1 & 16.5 \\
\hline$T E_{04}$ & 12.8 & 18.8 & 21.8 & 149 & 12.7 & 18.6 & 24.2 & 16.5 \\
\hline$T E_{12}$ & 46.6 & 46.6 & 15.0 & 15.0 & 46.8 & 45.0 & 16.2 & 16.2 \\
\hline $\mathrm{TE}_{13}$ & 29.2 & 29.2 & 15.0 & 15.0 & 28.9 & 29.5 & 16.1 & 16.4 \\
\hline$T E_{14}$ & 21.3 & 21.3 & 15.0 & 15.0 & 21.0 & 21.4 & 16.4 & 16.4 \\
\hline$T E_{15}$ & 16.8 & 16.8 & 15.0 & 15.0 & 16.8 & 16.8 & 16.1 & 16.7 \\
\hline
\end{tabular}

Table 2. Waist sizes obtained by gaussian optics and spot sizes obtained by numerical calculations of the Huygens equation. The values $w_{x 0^{\prime}}$ and $w_{z 0^{\prime}}$ are waist sizes at the flter and $w_{x 0^{\prime \prime}}$ and $w_{y 0^{\prime \prime}}$ are those at the waist, respectively. The values $w_{x^{\prime}}$ and $w_{z^{\prime}}$ are spot sizes at the filter and $w_{x^{\prime \prime}}$ and $w_{y^{\prime \prime}}$ are at the waist. 
(a)

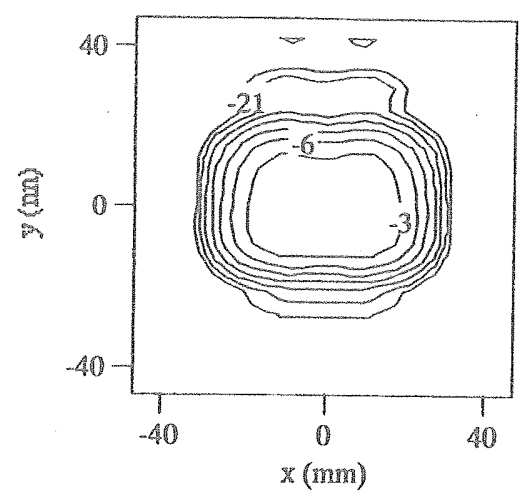

(c)

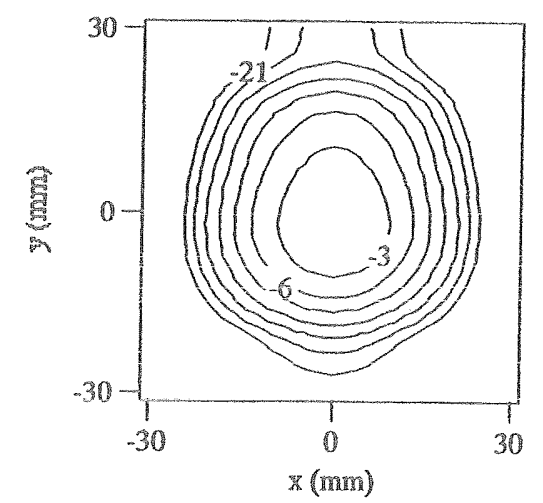

(b)

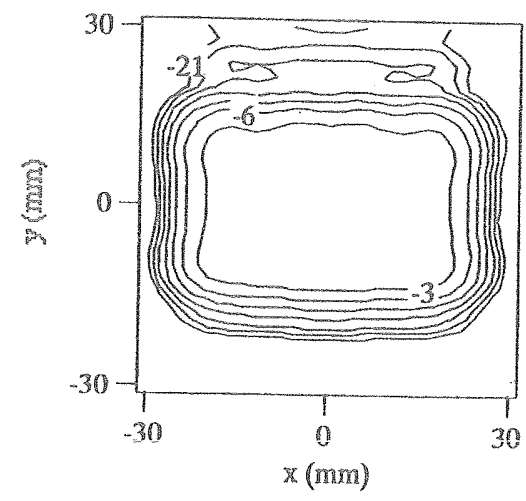

(d)

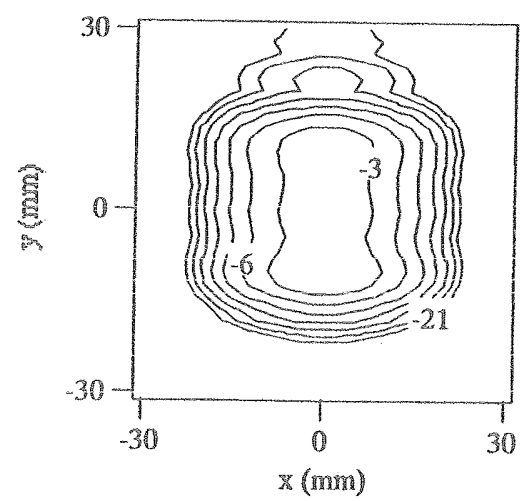

Figure 8. Calculated intensity contours at the beam waist without spatial filter: $(a) \mathrm{TE}_{02}$ mode $(223 \mathrm{GHz}) ;\left(\right.$ b) $\mathrm{TE}_{04}$ mode $(423 \mathrm{GHz}) ;\left(\right.$ c) $\mathrm{TE}_{12}$ mode $\left(170 \mathrm{GHz}\right.$ ); (d) $\mathrm{TE}_{15}$ mode $(472 \mathrm{GHz})$. Contours are in decibels relative to the intensity maximum.

patterns (see for example figure 7) the sidelobes are not symmetrical due to the oblique position of the effective image source with respect to the z-axis.

The intensity profiles at the $z=1.241$ plane are shown in figure 8 . Because their shapes are distorted by sidelobes seen in figures 6 and 7 , they are quite different from a gaussian beam or a bigaussian beam. In order to improve the quality of the beams produced, it is necessary to remove the sidelobes from the far-field of the image source. Experimentally this can be done by inserting a spatial filter to block the sidelobes in the $y=1.241$ plane.

In principle, several loss-less methods can be used to transform any field distribution to gaussian beams (Bogdashov et al. 1995). Unfortunately these techniques are not broad band. Alternatively, it is justifiable to sacrifice a small amount of the beam power in order to improve beam quality through truncation of the sidelobes by a spatial filter. The main advantage of the latter approach is that by its nature it ensures broad-band operation of the system. Additionally, this conceptually simple solution can be realized very easily in practice. 


\begin{tabular}{lccc}
\hline Mode & $\left(x_{c}, y_{c}, z_{c}\right)$ & $w_{x}(\mathrm{~mm})$ & $w_{z}(\mathrm{~mm})$ \\
\hline $\mathrm{TE}_{02}$ & $\left(0,1.241,-5 \times 10^{-3}\right)$ & 70 & 102 \\
$\mathrm{TE}_{03}$ & $\left(0,1.241,-3 \times 10^{-3}\right)$ & 50 & 70 \\
$T E_{04}$ & $\left(0,1.241,-2 \times 10^{-3}\right)$ & 40 & 56 \\
$T_{12}$ & $\left(0,1.241,-14 \times 10^{-3}\right)$ & 140 & 140 \\
$T_{13}$ & $\left(0,1.241,-6 \times 10^{-3}\right)$ & 87 & 87 \\
$T_{14}$ & $\left(0,1.241,-3 \times 10^{-3}\right)$ & 62 & 62 \\
$T_{15}$ & $\left(0,1.241,-4 \times 10^{-3}\right)$ & 52 & 52 \\
\hline
\end{tabular}

Table 3. The centre $\left(x_{c}, y_{c}, z_{c}\right)$ of the plane and the sizes $w_{x}, w_{z}$ in $x$ and $z$ directions for each mode.
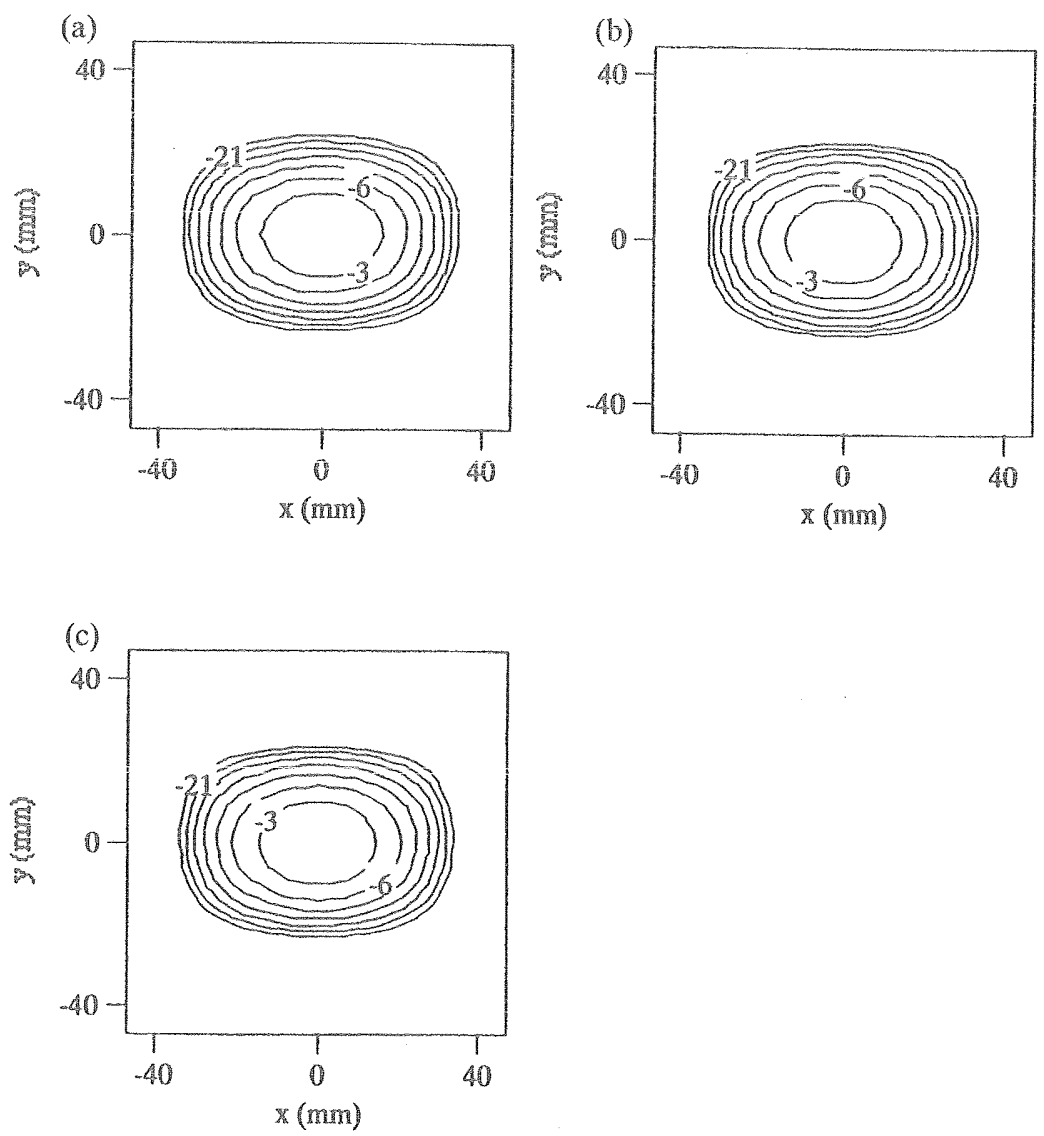

Figure 9. Calculated intensily contours at the output beam waist with spatial filter: $(a) \mathbb{T E}_{02}$ mode $(223 \mathrm{GH} z) ;(b) \mathrm{TE}_{03}$ mode $(323 \mathrm{GH} 7) ;(c) \mathrm{TE}_{04}$ mode $(423 \mathrm{GHz})$. Contours are in decibels relative to the intensity maximum. 
In our new conversion system the spatial filter used for removing the sidelobes is an indispensable component. It is just a thin plate with an aperture having dimensions $w_{x}, w_{z}$ and centre in $\left(x_{c}, y_{c}, z_{c}\right)$. The filter is located at the $y=1.241$ plane. From the calculated field patterns in this cross-section an appropriate position and sizes of the aperture are selected in such a way as to block efrectively only the sidelobes of the beam. More specifically, the aperture is selected large enough to encompass the $-21 \mathrm{~dB}$ contour of the main beam but small enough to truncate the $-21 \mathrm{~dB}$ contours of the sidelobes. The chosen parameters of the spatial filter are presented in table 3 . Then the electromagnetic fields on the subsequent mirror $m 2$ are calculated by using repeatedly the Huygens equation with fields at the aperture as sources.

The intensity profiles at the $z=1.241$ plane with spatial niltering for $\mathrm{TE}_{0 i}$ modes are shown in figure 9 and those for $\mathbb{T E}_{1 n}$ modes are shown in figures 10-13. One can

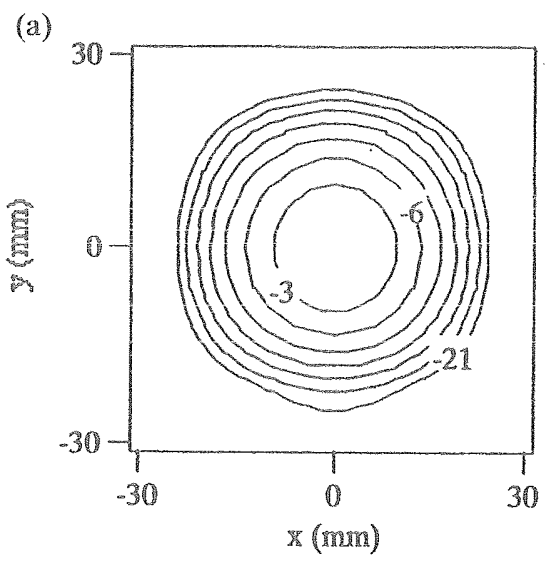

(b)

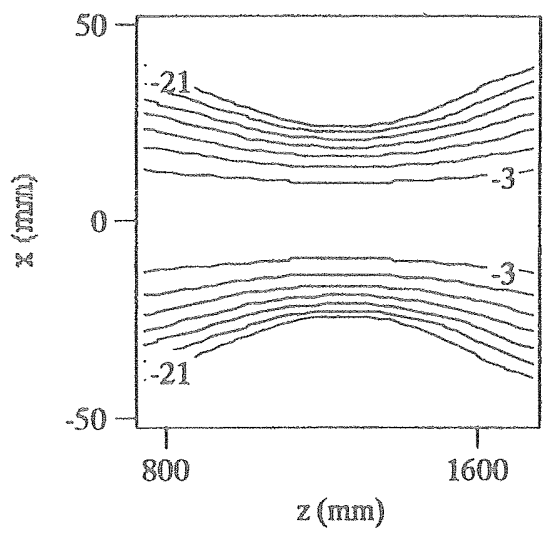

(c)

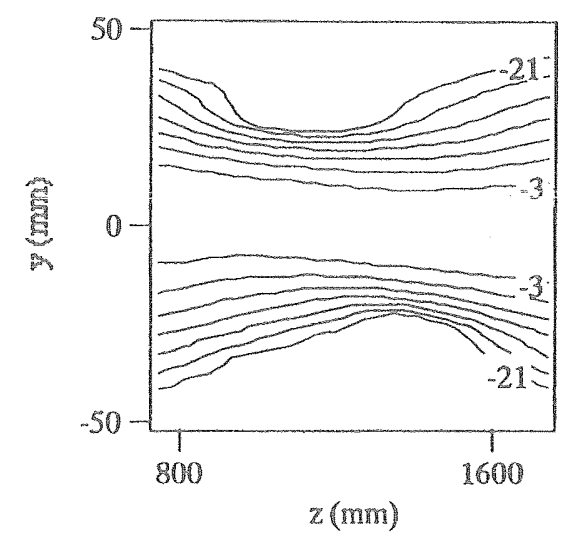

Figure 10. Calculated intensity contours for $\operatorname{TE}_{12}$ mode $(170 \mathrm{GHz})$ : (a) at the beam waist. Contours are in decibels relative to the intensity maximum; $(b)$ and $(c)$ in the vicinity of the beam waist. Contours are relative to the intensity along the $z$-axis. 

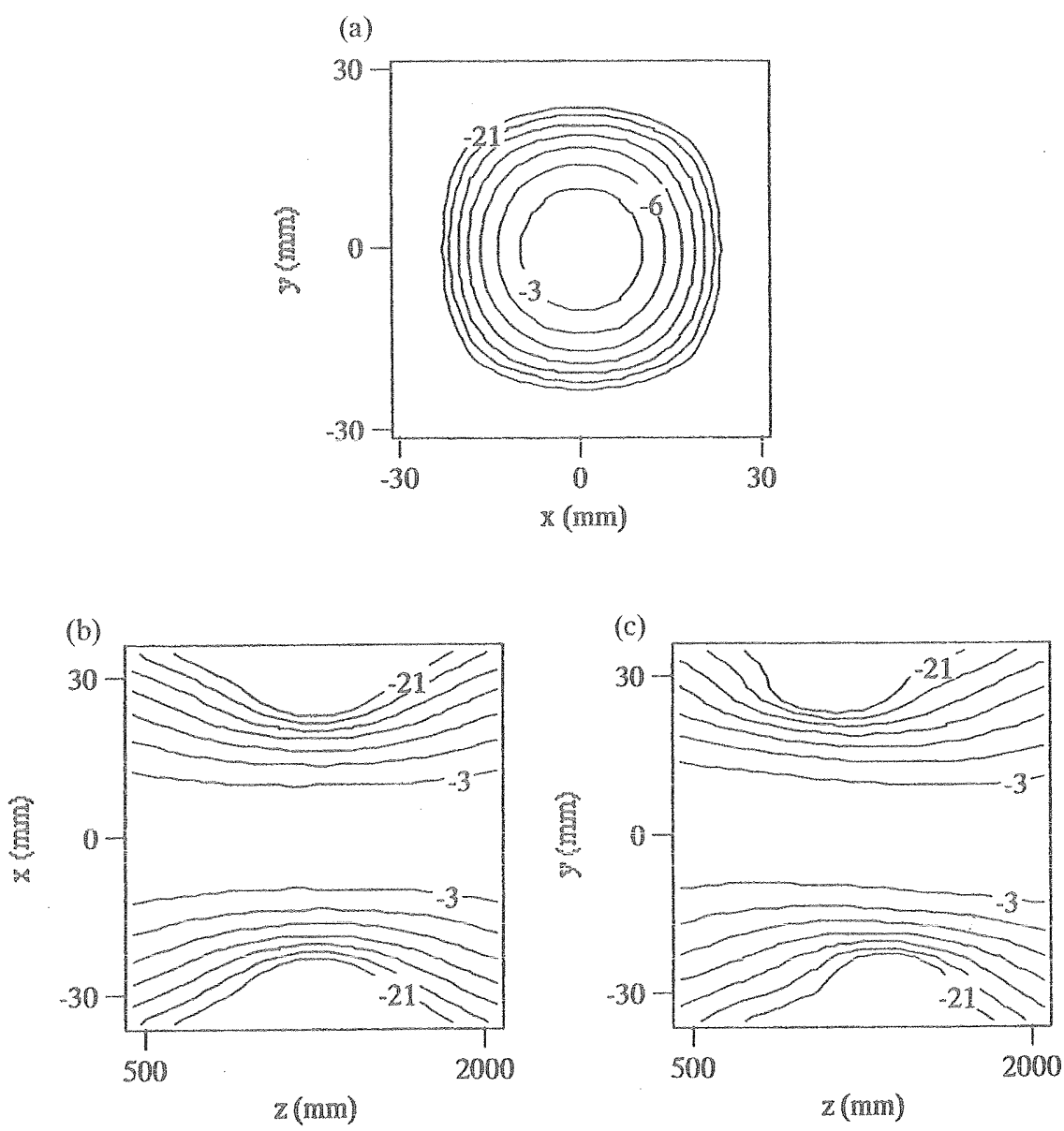

Figure 11. Calculated intensity contours for $\mathrm{TE}_{13}$ mode $(271 \mathrm{GHz}):(a)$ at the beam waist. Contours are in decibels relatve to the intensity naximum; $(b)$ and $(c)$ in the vicinity of the beam waist. Contours are relative to the intensity along the $z$-axis.

see that this system converts four $\mathrm{TE}_{1 n}$ mode outputs of the Gyrotron FU IVA into gaussian-like beams with waist size of $w_{x^{\prime \prime}}=w_{y^{\prime \prime}}=16.3 \mathrm{~mm}$ as well as three $T E_{0 n}$ mode outputs into bigaussian-like beams with waist size of $w_{x^{\prime \prime}}=23.5 \mathrm{~mm}$ and $w_{y 0^{\prime \prime}}=16.4 \mathrm{~mm}$. The spot sizes $w_{x^{\prime \prime}}$ and $w_{y^{\prime \prime}}$ agree well with the waist sizes $w_{x 0^{\prime \prime}}$ and $w_{y 0}$ " given by equation (6) (table 2).

Most of the power $\left(85 \%\right.$ for $\mathbb{T E}_{0 n}$ mode and $90 \%$ for $\mathbb{T E}_{1 n}$ mode) from the image source still arrives at the beam waist in spite of the truncation of the sidelobes at the $y=1.241$ plane. It should be noticed that loses for different $\mathrm{TE}_{1 n}$ modes differ insignificantly (less than $1 \%$ ). The same applies to the $\mathrm{TE}_{0 n}$ mode outputs.

\section{Conclusion}

The conversion system presented in this paper consists of a quasi-optical antenna, sequence of two ellipsoidal focusing mirrors and a spatial filter. It is 


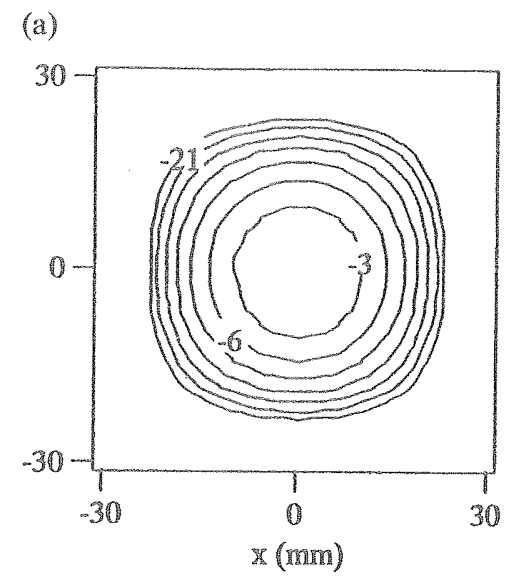

(b)

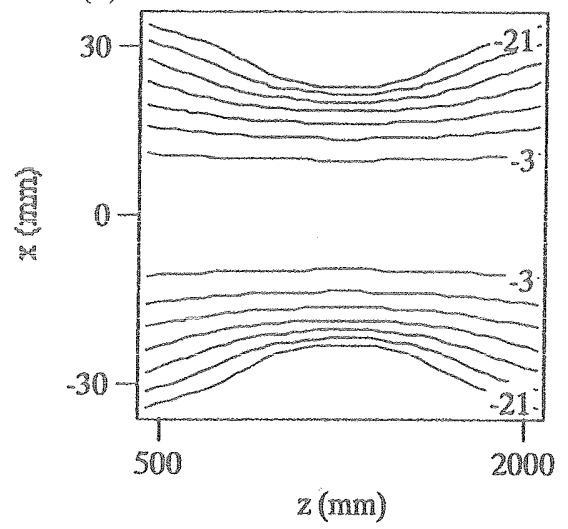

(c)

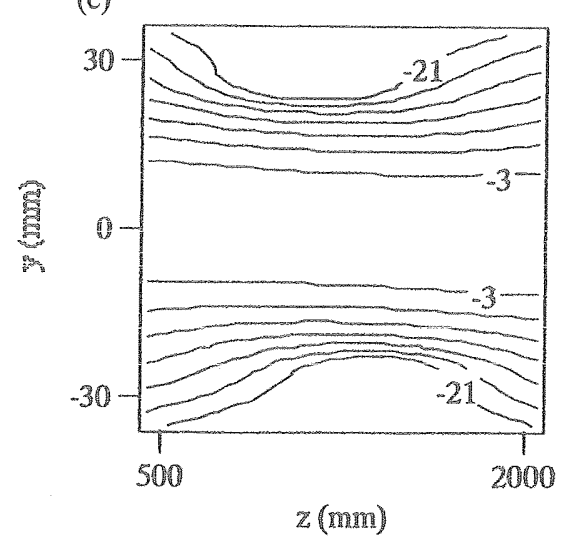

Figure 12. Calculated intensity contours for $\mathrm{TE}_{1}$ mode $(372 \mathrm{GHz})$ ( $a$ ) at the beam waist. Contours are in decibels relative to the intensity maximum; $(b)$ and $(c)$ in the vicinity of the beam waist. Contours are relative to the intensity along the $z$-axis.

intended to convert four $T_{1}$ mode outputs of the Gyrotron FU IVA $\left(T E_{12}\right.$, $170 \mathrm{GHz}_{2}, \mathrm{TE}_{13}, 271 \mathrm{GHz} ; \mathrm{TE}_{14}, 372 \mathrm{GHz} ; \mathrm{TE}_{15}, 472 \mathrm{GHZ}$ ) into gaussian-like beams with waist size of $w_{x^{\prime \prime}}=w_{y^{\prime \prime}}=16.3 \mathrm{~mm}$ as well as three $\mathbb{T E}_{0 n}$ modes $\left(\mathrm{TE}_{02}, 223 \mathrm{GHz} ; \mathrm{TE}_{03}, 323 \mathrm{GHz}_{2} \mathrm{TE}_{04}, 423 \mathrm{GHz}\right)$ into bigaussian-like beams with waist sizes of $w_{x^{\prime \prime}}=23.5 \mathrm{~mm}$ and $w_{y 0}=16.4 \mathrm{~mm}$. Compared with the earlier system capable of converting only one operating mode the novel one is more versatile and is compatible with frequency tunable gyrotrons such as the Gyrotron FU IVA. In spite of the truncation of the sidelobes of the beam by the spatial filter most of the image source power is transferred to the final waist. As a result, intense high-quality beams in the submillimetre wavelength range can be produced. 

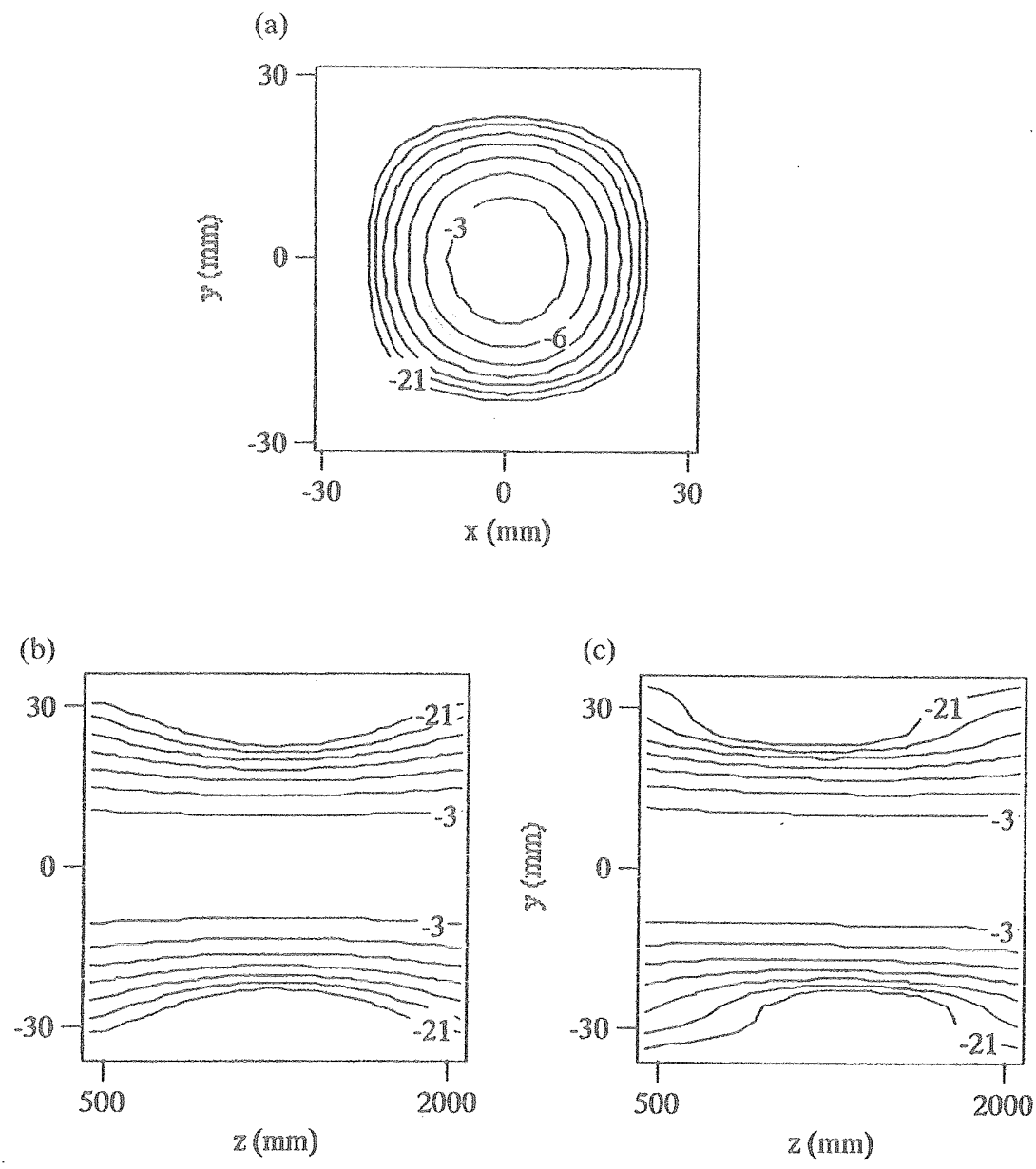

Figure 13. Calculated intensity contours for $\mathrm{TE}_{15}$ mode $(472 \mathrm{GH} / \mathrm{z})$ ) (a) at the beam waist. Contours are in decibels relative to the intensity maximum; $(b)$ and $(c)$ in the vicinity of the beam waist. Contours are relative to the intensity along the $z$-axis.

\section{Acknowledgments}

This work was done as a collaboration between the Fukui University and the University of Stuttgart, Germany and was partially supported by a Grant-in-Aid from the Ministry of Education, Science and Culture of Japan and the Murata Science Foundation.

Numerical calculations were made at the Computer Center of the National Institute for Fusion Science.

\section{References}

Bogdashov, A. A., Chirkov, A. V., Dinisov, G. G., Vinogradov, D. V., Kuftin, A. N., Malygin, V. I., and Zapevalov, V. E., 1995, Mirrors synthesis for gyrotron quasioptical mode converters. Intemational Jomal of Infrared and Millimeter Waves, 16 , 735-744. 
Brand, G. F., Pekete, R. W., Hong, K., MoORe, K. I., and ldehara, T, 1990 b, Operating of a tunable gyrotron at the second hamonic of the electron cyclotron rrequency. International Journal of Electronics, 68, 1099-1111.

Brand, G. F. Fekete, P. W., PDEHAara, T, and MoORE, K. J., 1990 a, Quasi-optical antennas for plasma scattering. International Joumal of Electronics, 68, 1063-1073.

FEkrte, P. W. BRAND, G. F., and IDEHARA, T, 1994, Scattering from discrete Alven waves in a tokamak using a gyrotron radiation source. Plasma Physics and Controlled Fusion, $36,1407-1417$

Flyagin, V.A., LuCHINI, A. G., and Nusinovich, G. S., 1983, Submillimeter-wave gyrotrons: theory and experiment. Intenational Jounal of hrared and Millimeter Waves, 4,629 637.

Inehara, T., Nishuda, N., Yoshida, K., OGawa, I., Tatsumawa, T., Wagner, D., Gantenden, G., KaspareK, W., and THumM, M., 1998, High frequency and high mode purity operations of Gyrotron FU IVA. International Joumal of Infrared and Millimeter Waves, 19, 919-930.

Idehara, T., OGawa, I., Mitsudo, S., Pereyaslavets, M., Nishida, N., and Yoshida, $\mathbb{K}$., 1999, Development of frequency tunable, medium power gyrotrons (Gyrotron GU Series) as submillimeter wave radiation sources. IEEE Transactions on Plosma Science, $27,340-354$.

Idehara, T., Shimizu, Y., IChikawa, K., MaKino, S., Shbutank, KK., Kurahashi, K., TATSUKAWA, T., OGAWA, I., OKAzAKr, Y., and OKaMoTO, T., 1995, Development of a medium power, submillimeter wave gyrotron using a $17 \mathrm{~T}$ superconducting magnet. Physics of Plasmas, 2, 3246-3248.

Kong, J. A., 1986, Electromagnetic Wave Theory (New York: Wiley and Sons).

KREISCher, K. E., and TEMKIN, R. J., 1987, Single-mode operation of a high-power, steptunable gyrotron. Physical Review Letters, $\$ 94,54-550$

Nus Novich, G.S., and READ, M. E., 1999, Theory of step-tunable gyrotrons operating at two cyclotron hamonics. IEEE Transactions on Plasma Science, 27, 335-362.

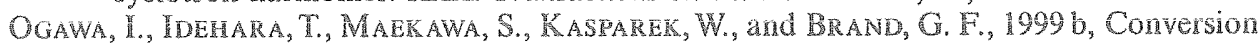
of gyotron output into a gaussian bean using the far-fid. International Joumal of Infrared and Millimeter Waves, 20, 801-821.

Ogawa, I., Inehara, T., Pereyaslavets, M., and Kasparex, W., 1999 a, Design of a quasioptical system converting the $T E_{06}$ output mode of a gyrotron into a gaussian-like beam. International Journal of Infrared and Millimeter Waves, 20, 543-558.

Ogawa, I., SaKal, A., IDEhakA, T, and KaSParek, W., $1999 \mathrm{c}$, Application of the complex beam parameter to the design of quasi-optical transmission line for a submillimeter

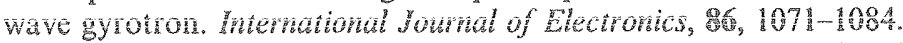

OGaWA, I., Sakal, A., IDEHARA, T., KaWAHATA, K., and KASparer, W., 1997, A quasi-optical transmission line for plasma scattering measurements using a submillimeter wave gyrotron. International Journal of Electronics, 83, 635-644.

Sibgman, A. E., 1971, An Iniroduction to Lasers and Masers (New York: McGraw-Hill).

Spira-HakKarainen, S., Kruiscier, K. E., and TEMKIN, R. J., 1990, Submillimeterwave harmonic gyrotron experiment. HEEE Transactions on Plasma Science, 18 , $334-342$.

Suvorov, E. V. Holzhauer, E., Kasparek, W., Lueyako, L. V., Burov, A. B., Dreyagin, Y. A., Fll'CHEnkov, S. E., Framan, A. A., Kukin, L. M., Kostrov, A. V., Ryndik, D. A., Sutanyuk, A. M., Skalyga, N. K., Smolyakova, O. B., Erckmann, V., Guist, T., KiCK, M., LAQUA, H., and Rust, M., 1997, Collective Thomson scattering at WA-AS. Plasma Physics and Controlled Fusion, 39, B337-B351.

Terumiche, Y, Kubo, S., Ando, A., Yanagmoto, Y., OGURa, K., Tanaka, H., Takahash, I. TONAl, I., Nakamura, M., Maekawa, T., Tanaka, S., and Idehara, T., 1984, Study of low frequency density fluctuations in the WT-2 tokamak by mm and submm wave scattering. 9th International Conference on Infrared and Millimeter Woves, Takarazuka, Japan (Tokyo: Japan Society of Applied Physics), pp. $411-412$.

VLASOV, S. N., and ORLOVA, 1, M., 1974, Quasioptical transformer which transforms the waves in a waveguide having a circular cross section into a highly directional wave beam. Radiofzika, 17, 115-119. 
WADA, O, and NAKAJIMA, M., 1986, Reffector antennas for electron cyclotron resonance heating of fusion plasma. Space Power, 6, 213-220.

Woskobornikow, P., CoHN, D. R., and TEMKIN, R. J., 1983, Application of advanced millimeter/far-infrared sources to collective Thomson scattering plasma diagnostics. International Joumal of infrared and Millimeter Waves, 4, 205-229.

Zaytsev, N. I., Pankratova, T. B., Petgin, M. I., and Flyagln, V. A., 1974, Millimeterand submillimeter-wave gyrotrons. Radio Engineering and Electronic Physics, 19, 103-107. 